\title{
Five Dimensional Rotating Black Hole in a Uniform Magnetic Field: The Gyromagnetic Ratio
}

\author{
A. N. Aliev \\ Feza Gürsey Institute, P.K. 6 Çengelköy, 81220 Istanbul, Turkey \\ Valeri P. Frolov \\ Theoretical Physics Institute, Department of Physics, \\ University of Alberta, Edmonton, Canada T6G 2J1
}

(Dated: October 31, 2018)

\begin{abstract}
In four dimensional general relativity, the fact that a Killing vector in a vacuum spacetime serves as a vector potential for a test Maxwell field provides one with an elegant way of describing the behaviour of electromagnetic fields near a rotating Kerr black hole immersed in a uniform magnetic field. We use a similar approach to examine the case of a five dimensional rotating black hole placed in a uniform magnetic field of configuration with bi-azimuthal symmetry, that is aligned with the angular momenta of the Myers-Perry spacetime. Assuming that the black hole may also possess a small electric charge we construct the 5-vector potential of the electromagnetic field in the Myers-Perry metric using its three commuting Killing vector fields. We show that, like its four dimensional counterparts, the five dimensional Myers-Perry black hole rotating in a uniform magnetic field produces an inductive potential difference between the event horizon and an infinitely distant surface. This potential difference is determined by a superposition of two independent Coulomb fields consistent with the two angular momenta of the black hole and two nonvanishing components of the magnetic field. We also show that a weakly charged rotating black hole in five dimensions possesses two independent magnetic dipole moments specified in terms of its electric charge, mass, and angular momentum parameters. We prove that a five dimensional weakly charged Myers-Perry black hole must have the value of the gyromagnetic ratio $g=3$.
\end{abstract}




\section{INTRODUCTION}

Black holes originally predicted in four dimensional general relativity have subsequently become an inseparable part of all higher dimensional gravity theories. The fundamental features of black holes in four dimensions, such as the equilibrium and uniqueness properties, quantum properties following from Hawking's effect of evaporation of microscopic black holes have revealed an intimate connection between spacetime geometry, quantum field theory and thermodynamics [1, 2, 3, 4] (see Refs. [5, 6, 7] for comprehensive reviews). Certainly, these properties of black holes might have played a crucial role in the analysis of dynamics of higher dimensional gravity theories, as well as in the compactification process. For instance, to test the novel predictions of superstring theory which, as believed, provides a consistent quantum theory of gravity in higher dimensions [8], microscopic black holes may serve as good theoretical laboratories. Therefore over the last years higher dimensional black holes have been widely considered as very interesting objects to be investigated in detail. Many interesting black hole solutions in various higher-dimensional theories can be found in [9].

On the other hand, the interest in higher dimensional black holes has got a new impetus after the advent of brane-world gravity theories [10, 11, 12, 13] (see also Refs. [14, 15]). The brane-world theories are built on the basic idea that the geometry of our physical Universe is indeed a "3-brane" - (3+1) dimensional hypersurface, embedded in fundamental higher dimensional space. The size of the extra spatial dimensions may be much larger than the convensional Planckian length $\left(\sim 10^{-33} \mathrm{~cm}\right)$. Thus, in contrast to the original KaluzaKlein's scenario, in the brane-world models the extra dimensions are supposed to manifest themselves as physical ones. One of the dramatic consequences of these models is that the fundamental scale of quantum gravity might become as low as the weak interaction scale (of the order of $\mathrm{TeV}$ ). This, in turn, raises the problem of $\mathrm{TeV}$-size black holes and the exciting signature of such mini black holes is that they can be directly probed in cosmic ray experiments or at future high energy colliders [16, 17]. It has also been argued that one can describe these black holes by the classical solutions of higher dimensional vacuum Einstein equations provided that the radius of the event horizon is much smaller than the size-scale of the extra dimensions. In light of all this, it is obvious that further knowledge of the special properties of black hole solutions in higher dimensional vacuum gravity is of great importance. 
The first black hole solution to the higher dimensional Einstein equations is the static and hyperspherically symmetric Schwarzschild-Tangherlini solution [18], which has been found a long time ago within the Kaluza-Klein programme of extension of four dimensional general relativity. Like, in four dimensions, one could expect the generalization of the static hyperspherical black hole solution to include rotational dynamics. In 1986 Myers and Perry discovered the exact solution of Einstein's equations describing such rotating black holes 19]. It is important that the Myers-Perry solution is supposed to be the most relevant to describe the "laboratory" black holes in high-energy experiments [17]. In this context some essential features of the Myers-Perry solution in five dimensions, such as the existence of a Killing tensor and the separability of variables in the Hamilton-Jacobi equations of motion, as well as quantum radiation from a five dimensional black hole were explored in [20, 21].

However, the Myers-Perry solution is not unique in five dimensions, unlike its four dimensional counterpart, the Kerr solution. Emparan and Reall [22] found a rotating black ring solution in five dimensios with the horizon of toplogy of $S^{2} \times S^{1}$, which could have the same mass and spin as the Myers-Perry solution. As for the static case, the authors of 23, 24] have proved that in this case, remarkably, the uniqueness property survives. Another essential problem is the stability of higher dimensional rotating black hole solutions. Recently in paper [25] it has been argued that the Myers-Perry solution becomes unstable for the case of an arbitrary large rotation parameter for a fixed mass. It is clear that, in the general case, the full analytic theory of perturbations of higher dimensional rotating black holes is needed to resolve the stabilitiy problem. In the static case the stability of higher dimensional black holes was proved in [26].

In this paper we shall study further properties of a five dimensional rotating black hole in the presence of an external magnetic field, which is supposed to be uniform at infinity. We shall consider the configuration of the magnetic field aligned with the angular momenta of the black hole. In other words, the magnetic field shares the bi-azimuthal symmetry of the Myers-Perry spacetime and has only two nonvanishing components. In order to construct the corresponding solution of the Maxwell field equations in the background of the MyersPerry metric, we shall appeal to the well known fact [27] that in a vacuum spacetime one can obtain the solution for the Maxwell test field by using only isometries of that spacetime. Earlier, this fact was used in four dimensional general relativity to construct the solution for the electromagnetic field around a Kerr black hole immersed in a uniform magnetic field [28]. 
In this analysis the temporal and axial Killing vectors of the Kerr spacetime were used as a vector potential for the Maxwell test field. In particular, it has been found that the so-called Wald effect takes place; the rotation of a black hole in an asymptotically uniform magnetic field causes an inductive potential difference between the event horizon and infinity, thereby the black hole may acquire an inductive electric charge.

In four dimensions the rotation group is $S O(3)$ and there always exists a rotation axis consistent with only one independent Casimir invariant. However, in five dimensions the rotation group is $S O(4)$, which posseses two independent Casimir invariants. These two Casimir invariants, in turn, are associated with two independent rotations of the system. In other words, a rotating black hole in five dimensions may have two distinct planes of rotation specified by appropriate azimuthal coordinates, rather than an axis of rotation. In accordance with this, the stationary and asymptotically flat Myers-Perry metric admits three commuting Killing vector fields, which reflect the time-translation invariance and biazimuthal symmetry of this metric in five dimensions. We shall use these Killing vectors to construct the 5-vector potential for a test electromagnetic field, when the Myers-Perry black hole is placed in an asymptotically uniform magnetic field of a configuration with bi-azimuthal symmetry. We shall show that a rotation in two distinct planes of a five dimensional black hole immersed in a uniform magnetic field produces an inductive electric field which is determined by the superposition of two independent Coulomb parts consistent with the two angular momentum parameters and two nonvanishing components of the magnetic field.

We shall also examine the case, when a five dimensional black hole may have an electric charge small enough that the spacetime is still well described by the Myers-Perry solution. In this case the rotation of the black hole must produce a dipole magnetic field. We shall establish that the black hole, in fact, possesses two independent magnetic dipole moments determined only by its charge, mass and angular momentum.

The paper is organized as follows. In Sec.II we recall some of the properties of the Myers-Perry metric for a five dimensional black hole. We describe the Killing isometries of the metric, its mass parameter, and specific angular momenta. We also give the precise definition of the angular velocities for stationary observers in the Myers-Perry metric and define locally non-rotating orthonormal frames (LNRFs) and their dual basis forms. In Sec.III we begin with a brief description of a uniform magnetic field in five dimensions and 
construct the 5-vector potential for the Maxwell test field using the Killing isometries of the Myers-Perry spacetime. Here we also calculate the magnetic flux crossing a portion of the black hole horizon. The dominant orthonormal components of electric and magnetic fields in the asymptotic rest frame of a weakly charged Myers-Perry black hole, as well as its magnetic dipole moments are calculated in Sec.IV . Finally, in Sec.V we prove that a five dimensional weakly charged Myers-Perry black hole must possess the value of the gyromagnetic ratio $g=3$.

\section{FIVE DIMENSIONAL ROTATING BLACK HOLE}

\section{A. Myers-Perry metric}

The metric of a rotating black hole in five dimensions follows from the general asymptotically flat solutions to $(N+1)$ dimensional vacuum gravity found by Myers and Perry 19]. In Boyer-Lindquist type coordinates it takes the most simple form given by

$$
\begin{aligned}
d s^{2}= & -d t^{2}+\Sigma\left(\frac{r^{2}}{\Delta} d r^{2}+d \theta^{2}\right)+\left(r^{2}+a^{2}\right) \sin ^{2} \theta d \phi^{2}+\left(r^{2}+b^{2}\right) \cos ^{2} \theta d \psi^{2} \\
& +\frac{m}{\Sigma}\left(d t-a \sin ^{2} \theta d \phi-b \cos ^{2} \theta d \psi\right)^{2}
\end{aligned}
$$

where

$$
\Sigma=r^{2}+a^{2} \cos ^{2} \theta+b^{2} \sin ^{2} \theta, \quad \Delta=\left(r^{2}+a^{2}\right)\left(r^{2}+b^{2}\right)-m r^{2}
$$

and $m$ is a parameter related to the physical mass of the black hole, while the parameters $a$ and $b$ are associated with its two independent angular momenta. For the metric determinant we have

$$
\sqrt{-g}=r \Sigma \sin \theta \cos \theta
$$

The components of the inverse metric have the following forms:

$$
\begin{aligned}
& g^{t t}=-\left(1+\frac{m}{\Sigma}+\frac{m r^{2}}{\Delta \Sigma}\right), \quad g^{r r}=\frac{\Delta}{r^{2} \Sigma}, \quad g^{\theta \theta}=\frac{1}{\Sigma}, \quad g^{\phi \psi}=-\frac{m a b}{\Delta \Sigma}, \\
& g^{t \phi}=-\frac{m a\left(r^{2}+b^{2}\right)}{\Delta \Sigma}, \quad g^{\phi \phi}=\frac{1}{\Sigma}\left[\frac{1}{\sin ^{2} \theta}+\frac{\left(r^{2}+b^{2}\right)\left(b^{2}-a^{2}\right)-m b^{2}}{\Delta}\right], \\
& g^{t \psi}=-\frac{m b\left(r^{2}+a^{2}\right)}{\Delta \Sigma}, \quad g^{\psi \psi}=\frac{1}{\Sigma}\left[\frac{1}{\cos ^{2} \theta}+\frac{\left(r^{2}+a^{2}\right)\left(a^{2}-b^{2}\right)-m a^{2}}{\Delta}\right] \text {, }
\end{aligned}
$$


The event horizon of the black hole is a null surface determined by the equation $g^{r r}=0$, which implies that

$$
\Delta=\left(r^{2}+a^{2}\right)\left(r^{2}+b^{2}\right)-m r^{2}=0
$$

The largest root of this equation gives the radius of the black hole's outer event horizon. We have

$$
r_{h}^{2}=\frac{1}{2}\left(m-a^{2}-b^{2}+\sqrt{\left(m-a^{2}-b^{2}\right)^{2}-4 a^{2} b^{2}}\right)
$$

Notice that the horizon exists if and only if

$$
a^{2}+b^{2}+2|a b| \leq m
$$

so that the condition $m=a^{2}+b^{2}+2|a b|$ or, equivalently, $r_{h}^{2}=|a b|$ defines the extremal horizon of a five dimensional black hole.

In the absence of the black hole $(m=0)$, the metric (11) reduces to the flat one written in oblate bi-polar coordinates. The latter can be readily cast in the Minkowski form using the transformation of coordinates [20]

$$
\begin{aligned}
& x=\sqrt{r^{2}+a^{2}} \sin \theta \cos \tilde{\phi}, \quad y=\sqrt{r^{2}+a^{2}} \sin \theta \sin \tilde{\phi} \\
& z=\sqrt{r^{2}+b^{2}} \cos \theta \cos \tilde{\psi}, \quad w=\sqrt{r^{2}+b^{2}} \cos \theta \sin \tilde{\psi}
\end{aligned}
$$

where

$$
\tilde{\phi}=\phi+\tan ^{-1}(a / r) \quad \tilde{\psi}=\psi+\tan ^{-1}(b / r)
$$

On the other hand, for $a=b=0$ we have the Schwarzschild-Tangherlini static solution in spherical bi-polar coordinates. It is clear that in general, the metric (11) admits two orthogonal 2-planes of rotation ( $\mathrm{x}-\mathrm{y}$ plane, $\mathrm{z}=\mathrm{w}=0)$ and $(\mathrm{z}-\mathrm{w}$ plane, $\mathrm{x}=\mathrm{y}=0$ ), which are specified by the azimuthal angles $\phi$ and $\psi$ respectively. These angles both range from 0 to $2 \pi$, while $\theta$ being the angle between the two orthogonal 2-planes varies in the interval $[0, \pi / 2]$. As a consequence, the metric reveals the following obvious invariance properties; under the simultaneous inversion of the time coordinate $t \rightarrow-t$ and the angles $\phi \rightarrow-\phi$, $\psi \rightarrow-\psi$, and under the transformation

$$
a \leftrightarrow b, \quad \phi \leftrightarrow \psi, \quad \theta \leftrightarrow \frac{\pi}{2}-\theta
$$


The bi-azimuthal symmetry properties of the five dimensional black hole metric (11) along with its stationarity imply the existence of the three commuting Killing vectors

$$
\xi_{(t)}=\partial / \partial t, \quad \xi_{(\phi)}=\partial / \partial \phi, \quad \xi_{(\psi)}=\partial / \partial \psi
$$

The various scalar products of these Killing vectors are expressed through the corresponding metric components as follows

$$
\begin{array}{ll}
\xi_{(t)} \cdot \xi_{(t)}=g_{t t}=-1+\frac{m}{\Sigma}, & \xi_{(\phi)} \cdot \xi_{(\phi)}=g_{\phi \phi}=\left(r^{2}+a^{2}+\frac{m a^{2}}{\Sigma} \sin ^{2} \theta\right) \sin ^{2} \theta \\
\xi_{(t)} \cdot \xi_{(\phi)}=g_{t \phi}=-\frac{m a}{\Sigma} \sin ^{2} \theta, & \xi_{(\psi)} \cdot \xi_{(\psi)}=g_{\psi \psi}=\left(r^{2}+b^{2}+\frac{m b^{2}}{\Sigma} \cos ^{2} \theta\right) \cos ^{2} \theta \\
\xi_{(t)} \cdot \xi_{(\psi)}=g_{t \psi}=-\frac{m b}{\Sigma} \cos ^{2} \theta, & \xi_{(\phi)} \cdot \xi_{(\psi)}=g_{\phi \psi}=\frac{m a b}{\Sigma} \sin ^{2} \theta \cos ^{2} \theta
\end{array}
$$

The Killing vectors (10) can be used to give a physical interpretation of the parameters $m, a$ and $b$ involved in the metric (11). Namely, following the analysis given in [29] one can obtain coordinate-independent definitions for these parameters. We have the integrals

$$
m=\frac{1}{4 \pi^{2}} \oint \xi_{(t)}^{\mu ; \nu} d^{3} \Sigma_{\mu \nu}
$$

and

$$
j_{(a)}=a m=-\frac{1}{4 \pi^{2}} \oint \xi_{(\phi)}^{\mu ; \nu} d^{3} \Sigma_{\mu \nu}, \quad j_{(b)}=b m=-\frac{1}{4 \pi^{2}} \oint \xi_{(\psi)}^{\mu ; \nu} d^{3} \Sigma_{\mu \nu}
$$

where the integrals are taken over the 3 -sphere at spatial infinity,

$$
d^{3} \Sigma_{\mu \nu}=\frac{1}{3 !} \sqrt{-g} \epsilon_{\mu \nu \alpha \beta \gamma} d x^{\alpha} \wedge d x^{\beta} \wedge d x^{\gamma},
$$

the semicolon denotes covariant differentiation and we have introduced the two specific angular momentum parameters $j_{(a)}$ and $j_{(b)}$ associated with rotations in the $\phi$ and $\psi$ directions respectively. We note that with these definitions the relation between the specific angular momentum and the mass parameter looks exactly like the corresponding relation $(J=a M)$ of four dimensional Kerr metric.

To show that the definitions given in (12) and (13) do in fact correctly describe the mass and angular momenta parameters we can calculate the integrands in the asymptotic region $r \rightarrow \infty$. The dominant terms in the asymptotic expansion have the form

$$
\begin{aligned}
& \xi_{(t)}^{t ; r}=\frac{m}{r^{3}}+\mathcal{O}\left(\frac{1}{r^{5}}\right), \\
& \xi_{(\phi)}^{t ; r}=-\frac{2 a m \sin ^{2} \theta}{r^{3}}+\mathcal{O}\left(\frac{1}{r^{5}}\right), \quad \xi_{(\psi)}^{t ; r}=-\frac{2 b m \cos ^{2} \theta}{r^{3}}+\mathcal{O}\left(\frac{1}{r^{5}}\right) .
\end{aligned}
$$


The substitution of these expressions into the formulas (12) and (13) verifies them. On the other hand, the relation of the above parameters to the total mass $M$ and the total angular momenta $J_{(a)}$ and $J_{(b)}$ of the black hole can be established using the formulas given in [19]. We obtain that

$$
m=\frac{8}{3 \pi} M, \quad j_{(a)}=\frac{4}{\pi} J_{(a)}, \quad j_{(b)}=\frac{4}{\pi} J_{(b)} .
$$

These relations confirm the interpretation of the parameters $m, a$ and $b$ as being related to the physical mass and angular momenta of the metric (11).

\section{B. Locally Nonrotating Observers}

To examine further properties of the five dimensional Myers-Perry black hole, as well as physical processes near such a black hole, it is useful to introduce a family of locally nonrotating observers. In the four-dimensional case a locally nonrotating observer has a vector of velocity orthogonal to the $t=$ const surface in the Kerr geometry and its angular

momentum vanishes [30, 31]. We define a locally nonrotating observer in the Myers-Perry metric in a similar manner. Let us write

$$
u^{\mu}=u^{\mu}(r, \theta)=\alpha\left(\xi_{(t)}^{\mu}+\Omega_{(a)} \xi_{(\phi)}^{\mu}+\Omega_{(b)} \xi_{(\psi)}^{\mu}\right)
$$

where $u^{\mu}$ is a unit vector of 5 -velocity of a locally nonrotating observer, and $\alpha$ is a normalization constant determined by the condition $u^{2}=-1$. The orthogonality to the $t=$ const surface implies $u^{r}=u^{\theta}=0$ and

$$
\begin{aligned}
g_{t \phi} u^{t}+g_{\phi \phi} u^{\phi}+g_{\phi \psi} u^{\psi} & =0, \\
g_{t \psi} u^{t}+g_{\psi \psi} u^{\psi}+g_{\psi \phi} u^{\phi} & =0 .
\end{aligned}
$$

The simultaneous solution of these equations determines $u^{\mu}(r, \theta)$. Thus we obtain

$$
\begin{aligned}
& \Omega_{(a)}=\frac{u^{\phi}}{u^{t}}=\frac{g_{t \psi} g_{\phi \psi}-g_{t \phi} g_{\psi \psi}}{g_{\phi \phi} g_{\psi \psi}-g_{\phi \psi}{ }^{2}}=\frac{a m\left(r^{2}+b^{2}\right)}{\Delta \Sigma+m\left(r^{2}+a^{2}\right)\left(r^{2}+b^{2}\right)}, \\
& \Omega_{(b)}=\frac{u^{\psi}}{u^{t}}=\frac{g_{t \phi} g_{\phi \psi}-g_{t \psi} g_{\phi \phi}}{g_{\phi \phi} g_{\psi \psi}-g_{\phi \psi}{ }^{2}}=\frac{b m\left(r^{2}+a^{2}\right)}{\Delta \Sigma+m\left(r^{2}+a^{2}\right)\left(r^{2}+b^{2}\right)} .
\end{aligned}
$$

In the case that either $b=0$, or $a=0$ instead of the above expressions we have either

$$
\Omega_{(a)}=-\frac{g_{t \phi}}{g_{\phi \phi}}, \text { or } \quad \Omega_{(b)}=-\frac{g_{t \psi}}{g_{\psi \psi}}
$$


which are closly reminiscent of the corresponding relation for the Kerr black hole in four dimensions. At large distances from the black hole, the relations (19) can be written in the form

$$
\Omega_{(a)}=\frac{j_{(a)}}{r^{4}}+\mathcal{O}\left(\frac{1}{r^{6}}\right), \quad \Omega_{(b)}=\frac{j_{(b)}}{r^{4}}+\mathcal{O}\left(\frac{1}{r^{6}}\right)
$$

which reveals the remarkable property of the Myers-Perry black hole, namely the dragging of inertial frames in both $\phi$ and $\psi$ 2-planes of rotation. Clearly, the effect of "bi-dragging" disappears at spatial infinity. However, toward the event horizon of the black hole it increases tending to its constant value on the event horizon $(\Delta=0)$. From equations (19) we obtain

$$
\Omega_{(a) h}=\frac{a}{r_{+}^{2}+a^{2}}, \quad \Omega_{(b) h}=\frac{b}{r_{+}^{2}+b^{2}} .
$$

These quantities can be interpreted as angular velocities of the black hole [19]. In order to show this, one needs to know the isometry properties of the horizon geometry. Following the Hawking approach in four dimensional geometry of the Kerr black hole [3], we suppose that the isometry of the event horizon of a five dimensional black hole is described by a Killing vector, which must be a linear combination of the three Killing vectors given in (10). Thus, we can take it in the form

$$
\chi=\xi_{(t)}+\Omega_{(a) h} \xi_{(\phi)}+\Omega_{(b) h} \xi_{(\psi)} .
$$

One can easily verify that this vector becomes null at the surface $\Delta=0$, i.e. it is tangent to the null surface of the horizon. This means that stationary observers moving in the $\phi$ and $\psi 2$-planes of rotation become co-rotating along with the horizon with the local angular velocities (22). In other words, the event horizon of the five dimensional Myers-Perry black hole is, in fact, the Killing horizon determined by the vector $\chi$.

From what we said above about the locally nonrotating observers it also follows that with any such observer one can associate an orthonormal frame. The form of the metric given in (11) enables us to choose the appropriate basis 1-forms of these frames. In particular, for a locally nonrotating observer moving in the $\phi 2$-plane we can choose an orthonormal frame $(L N R F)$ with the following basis 1-forms 


$$
\begin{aligned}
\omega^{\hat{t}} & =\left|g_{t t}-\frac{g_{t \psi}{ }^{2}}{g_{\psi \psi}}-\Omega_{(a)}^{2} \frac{g_{\phi \phi} g_{\psi \psi}-g_{\phi \psi}{ }^{2}}{g_{\psi \psi}}\right|^{1 / 2} d t \\
\omega^{\hat{\phi}} & =\left(\frac{g_{\phi \phi} g_{\psi \psi}-g_{\phi \psi}{ }^{2}}{g_{\psi \psi}}\right)^{1 / 2}\left(d \phi-\Omega_{(a)} d t\right) \\
\omega^{\hat{\psi}} & =\left(g_{\psi \psi}\right)^{1 / 2}\left(d \psi+\frac{g_{t \psi}}{g_{\psi \psi}} d t+\frac{g_{\phi \psi}}{g_{\psi \psi}} d \phi\right) \\
\omega^{\hat{r}} & =\left(g_{r r}\right)^{1 / 2} d r \\
\omega^{\hat{\theta}} & =\left(g_{\theta \theta}\right)^{1 / 2} d \theta,
\end{aligned}
$$

while the dual basis is given by

$$
\begin{aligned}
e_{\hat{t}} & =\left|g_{t t}-\frac{g_{t \psi}{ }^{2}}{g_{\psi \psi}}-\Omega_{(a)}^{2} \frac{g_{\phi \phi} g_{\psi \psi}-g_{\phi \psi}^{2}}{g_{\psi \psi}}\right|^{-1 / 2}\left(\frac{\partial}{\partial t}+\Omega_{(a)} \frac{\partial}{\partial \phi}-\frac{g_{t \psi}+\Omega_{(a)} g_{\phi \psi}}{g_{\psi \psi}} \frac{\partial}{\partial \psi}\right) \\
e_{\hat{\phi}} & =\left(\frac{g_{\phi \phi} g_{\psi \psi}-g_{\phi \psi}^{2}}{g_{\psi \psi}}\right)^{-1 / 2}\left(\frac{\partial}{\partial \phi}-\frac{g_{\phi \psi}}{g_{\psi \psi}} \frac{\partial}{\partial \psi}\right) \\
e_{\hat{\psi}} & =\frac{1}{\left(g_{\psi \psi}\right)^{1 / 2}} \frac{\partial}{\partial \psi} \\
e_{\hat{r}} & =\frac{1}{\left(g_{r r}\right)^{1 / 2}} \frac{\partial}{\partial r} \\
e_{\hat{\theta}} & =\frac{1}{\left(g_{\theta \theta}\right)^{1 / 2}} \frac{\partial}{\partial \theta}
\end{aligned}
$$

We note that the corresponding basis 1-forms and their duals associated with an orthonormal frame of a locally nonrotating observer moving in the $\psi$ direction are obtained from the expressions given above simply by the transformation $\phi \leftrightarrow \psi$.

\section{UNIFORM MAGNETIC FIELD IN THE BACKGROUND OF A FIVE DIMENSIONAL BLACK HOLE}

\section{A. Uniform magnetic field in a five dimensional flat spacetime}

In four dimensional gravity the behaviour of electromagnetic fields in the background of a rotating black hole described by the Kerr metric has been investigated by many authors 
[28, 32, 33, 34] (see also Ref. 35] for a review). In particular, Wald has proposed the most elegant way of desribing the behaviour of electromagnetic fields near a rotating black hole which is placed in an originally uniform magnetic field aligned with the axis of symmetry of the black hole [28]. The Wald approach stems from the well known fact 27] that a Killing vector in a vacuum spacetime serves as a vector potential for a Maxwell test field in that spacetime's background. Therefore one can construct a solution for the Maxwell test field in the background of a vacuum spacetime simply by using the isometries of this spacetime.

We shall apply this approach to examine the behaviour of a Maxwell test field around a five dimensional black hole described by the metric (11) when the black hole is immersed in an asymptotically uniform magnetic field. We recall that in general, the electromagnetic field in five dimensions can be described by an electric 1-form field and magnetic 2-form field. In other words, four of the total ten independent components of the electromagnetic field tensor $F_{\mu \nu}$ describe the electric field, while the remaining six components correspond to the magnetic field. We shall consider the case of a magnetic field configuration which is stationary and uniform at infinity and possesses bi-azimuthal symmetry as well. Then it is clear that the corresponding electromagnetic field tensor must share all isometries of the black hole's spacetime. On these grounds, it is also clear that there must exist only two nonvanishing components of the field tensor that describe a uniform magnetic field in our case. That is, we have

$$
B=F_{x y}, \quad H=F_{z w},
$$

where we have used the notations $B$ and $H$ for the magnetic field strengths associated with the $(\mathrm{x}-\mathrm{y})$ and $(\mathrm{z}-\mathrm{w})$ 2-planes, respectively. We note that these quantites are reminiscent of the two independent angular momenta given in (13), which are, in turn, the nonvanishing components of the underlying angular momentum 2-form. In this respect, the magnetic field components in our model are aligned with the corresponding angular momenta.

In the following we shall need the expression for the components of the electromagnetic 2-form field $F$ written down in bi-polar coordinates. By making use of the transformations (8) we obtain, instead of (26), an expression of the form

$$
\begin{aligned}
F= & B r \sin \theta(\sin \theta d r \wedge d \phi+r \cos \theta d \theta \wedge d \phi) \\
& +H r \cos \theta(\cos \theta d r \wedge d \psi-r \sin \theta d \theta \wedge d \psi),
\end{aligned}
$$


which describes a uniform magnetic field of a configuration with bi-azimuthal symmetry in a flat five dimensional spacetime.

\section{B. 5-Vector Potential}

It is remarkable that using only the isometries of the Myers-Perry spacetime described by the temporal Killing vector $\xi_{(t)}$ and two azimuthal Killing vectors $\xi_{(\phi)}$ and $\xi_{(\psi)}$ one can construct 5-vector potential for the Maxwell test field in this spacetime. Indeed, the homogeneous Maxwell equations, in the Lorentz gauge

$$
A_{; \mu}^{\mu}=0
$$

have the form

$$
A^{\mu ; \nu} ; \nu-R_{\nu}^{\mu} A^{\nu}=0
$$

On the other hand, any Killing vector $\xi$ satisfies the equation

$$
\xi_{; \nu}^{\mu ; \nu}+R_{\nu}^{\mu} \xi^{\nu}=0
$$

Comparing the two equations (29) and (30) one sees that they are the same in a vacuum spacetime $\left(R_{\nu}^{\mu}=0\right)$. Thus, one can use a Killing vector as a vector potential for a test Maxwell field [27]. Following this fact, we shall seek for a 5-vector potential of the form

$$
A^{\mu}=\alpha \xi_{(t)}^{\mu}+\beta \xi_{(\phi)}^{\mu}+\gamma \xi_{(\psi)}^{\mu}
$$

where $\alpha, \beta$ and $\gamma$ are arbitrary parameters. Let us emphasize that in a general case this vector potential will also describe a test electric field so that the black hole is charged. We assume that this electric charge is small enough, $Q \ll M$, so that the spacetime can still be adequately described by the unperturbed metric (11).

To determine the unknown parameters in (31) first we calculate the corresponding electromagnetic 2-form field $F$ in the metric (1). We obtain

$$
\begin{aligned}
F= & \frac{2 m r}{\Sigma^{2}} \mathcal{A} d t \wedge d r \\
& +\frac{m \sin 2 \theta}{\Sigma^{2}}\left[\alpha\left(b^{2}-a^{2}\right)+\beta a\left(r^{2}+a^{2}\right)-\gamma b\left(r^{2}+b^{2}\right)\right] d t \wedge d \theta \\
& +2 r \sin ^{2} \theta\left(\beta+\frac{a m}{\Sigma^{2}} \mathcal{A}\right) d r \wedge d \phi+2 r \cos ^{2} \theta\left(\gamma+\frac{b m}{\Sigma^{2}} \mathcal{A}\right) d r \wedge d \psi
\end{aligned}
$$




$$
\begin{aligned}
& +\sin 2 \theta\left[\beta\left(r^{2}+a^{2}\right)+\frac{a m}{\Sigma^{2}}\left(\mathcal{B}-\alpha\left(r^{2}+a^{2}\right)-\gamma b\left(r^{2}+b^{2}\right)\right)\right] d \theta \wedge d \phi \\
& -\sin 2 \theta\left[\gamma\left(r^{2}+b^{2}\right)+\frac{b m}{\Sigma^{2}}\left(\mathcal{B}-\alpha\left(r^{2}+b^{2}\right)-\beta a\left(r^{2}+a^{2}\right)\right)\right] d \theta \wedge d \psi,
\end{aligned}
$$

where for the sake of brevity we have used the notations

$$
\mathcal{A}=\alpha-\beta a \sin ^{2} \theta-\gamma b \cos ^{2} \theta
$$

and

$$
\mathcal{B}=\beta a\left(r^{2}+a^{2}+\Sigma\right) \sin ^{2} \theta+\gamma b\left(r^{2}+b^{2}+\Sigma\right) \cos ^{2} \theta
$$

In the asymptotic region $r \rightarrow \infty$, this expression takes the form

$$
\begin{aligned}
F= & 2 \beta r \sin \theta(\sin \theta d r \wedge d \phi+r \cos \theta d \theta \wedge d \phi) \\
& +2 \gamma r \cos \theta(\cos \theta d r \wedge d \psi-r \sin \theta d \theta \wedge d \psi)
\end{aligned}
$$

From a comparison of equations (27) and (35) it follows that $\beta=B / 2, \gamma=H / 2$. The remaining parameter $\alpha$ can be determined from examining the integrals (12) and (13) along with the integral for the electric charge of the black hole

$$
Q=\frac{1}{4 \pi^{2}} \oint F^{\mu \nu} d^{3} \Sigma_{\mu \nu}
$$

Having done this, we obtain

$$
\alpha=-\frac{Q}{2 m}+\frac{a B}{2}+\frac{b H}{2} .
$$

Finally, the 5-vector potential for the electromagnetic field around a rotating and weakly charged black hole in a uniform magnetic field can be written in the form

$$
A^{\mu}=-\frac{Q}{2 m} \xi_{(t)}^{\mu}+\frac{B}{2}\left(\xi_{(\phi)}^{\mu}+a \xi_{(t)}^{\mu}\right)+\frac{H}{2}\left(\xi_{(\psi)}^{\mu}+b \xi_{(t)}^{\mu}\right)
$$

From this expression, it follows that the 5-vector potential in the background of the MyersPerry spacetime consists of a superposition of the Coulomb-type part and the asymptotically uniform magnetic field. It is important to note that the Coulomb-type part, which is generated by the temporal Killing vector, does not vanish even when the electric charge of the black hole is zero $(Q=0)$. There are two independent contributions to the Coulomb field of the black hole. The physical reason underlying this phenomenon is that a rotation of a five dimensional black hole in a uniform magnetic field produces an inductive electric field 
associated with the two independent $\phi$ and $\psi$ 2-planes of rotation of the black hole as well as two independent components of the electromagnetic field tensor. It is seen that in our model the black hole acts like a "dynamo" that causes an electrostatic potential difference between the event horizon of the black hole and an infinitely distant surface. Following the approach in four dimensional case [36] we shall define the electrostatic potential of the event horizon with respect to the Killing vector (23) as follows

$$
\Phi_{h}=A \cdot \chi=A_{0}+\Omega_{(a) h} A_{\phi}+\Omega_{(b) h} A_{\psi} .
$$

Then, for the electrostatic potential difference between the event horizon and an infinitely distant surface, we find

$$
\triangle \Phi=\Phi_{h}-\Phi_{\infty}=\frac{Q-a m B-b m H}{2 m}
$$

This potential difference is exactly of the same form as if it were be produced by the electric charge

$$
\tilde{Q}=Q-a m B-b m H
$$

It is obvious that this charge $\tilde{Q}$ will be quickly neutralized (the potential difference vanishes) due to a selective accretion process of charged particles provided that the black hole is surrounded by an ionized medium 42] As a result of this the black hole will acquire the physical electric charge

$$
Q=a m B+b m H=j_{(a)} B+j_{(b)} H
$$

We note that in the degenerate case when $a=b$ and $B=H$ the above expression goes over into the form

$$
Q=2 a m B
$$

which is reminiscent of its counterpart for a Kerr black hole [28].

Accordingly, in terms of the total mass and angular momenta of the black hole the equation (42) can be written as

$$
Q=\frac{8}{3 \pi}(a M B+b m H)=\frac{4}{\pi}\left(J_{(a)} B+J_{(b)} H\right)
$$

Thus, a five dimensional black hole rotating in a uniform magnetic field of configuration with bi-azimuthal symmetry will charged up to the value given by equation (44). 
To conclude this section we shall calculate the magnetic flux crossing a portion $\Sigma$ of the black hole event horizon. This flux is governed by the line integral on the horizon

$$
\mathcal{F}=\int_{\partial \Sigma} A
$$

where the potential 1-form $A$ is determined through (38) with $Q=0$ and $\partial \Sigma$ is the boundary of $\Sigma$. For our purpose, it is convenient to rewrite the potential in terms of the Killing vector $\chi$ defined in Eq. (23). We obtain

$$
\begin{aligned}
& A=\frac{1}{2}(a B+b H) \chi+\tilde{A}, \\
& \tilde{A}=\frac{B}{2}\left(\xi_{(\phi)}-a \Omega_{(a) h} \xi_{(\phi)}-a \Omega_{(b) h} \xi_{(\psi)}\right)+\frac{H}{2}\left(\xi_{(\psi)}-b \Omega_{(b) h} \xi_{(\psi)}-b \Omega_{(a) h} \xi_{(\phi)}\right),
\end{aligned}
$$

where we have used the same notation for Killing 1-form fields. The first term in this expression is proportional to $\chi$ and therefore its contribution to the flux $\mathcal{F}$ at the horizon vanishes. For the rest part, taking into account (22) we obtain

$$
\tilde{A}=\frac{1}{2}\left(\frac{B r_{h}^{2}-H a b}{r_{h}^{2}+a^{2}} \xi_{(\phi)}+\frac{H r_{h}^{2}-B a b}{r_{h}^{2}+b^{2}} \xi_{(\psi)}\right) .
$$

This expression, and hence, the flux (45) vanishes precisely at the extremal horizon of the black hole $\left(r_{h}^{2}=a b\right)$, provided that $B=H$. Thus, when the magnetic field strengths associated with the (x-y) and (z-w) 2-planes of rotation are equal in magnitude, the magnetic

flux is expelled from a five dimensional black hole as the extremality in its rotation is approached. In this case, a portion of the black hole horizon, like its four dimensional counterpart, acts as the surface of a perfectly diamagnetic object [37].

\section{MAGNETIC DIPOLE MOMENTS}

We now turn to the consideration of a five dimensional weakly charged rotating black hole. It is clear that the rotation of such a black hole must produce a dipole type magnetic field around itself. Since the charged black hole is characterized by two independent rotation parameters, accordingly, one may expect that it will acquire two independent magnetic dipole moments as well. We shall determine the value of these magnetic moments.

We begin with the expression for the electromagnetic 2-form field 


$$
\begin{aligned}
F= & \frac{Q}{\Sigma^{2}}\left(r d r \wedge d t+\left(b^{2}-a^{2}\right) \sin \theta \cos \theta d \theta \wedge d t\right) \\
& -\frac{Q a \sin \theta}{\Sigma^{2}}\left(r \sin \theta d r \wedge d \phi-\left(r^{2}+a^{2}\right) \cos \theta d \theta \wedge d \phi\right) \\
& -\frac{Q b \cos \theta}{\Sigma^{2}}\left(r \cos \theta d r \wedge d \psi+\left(r^{2}+b^{2}\right) \sin \theta d \theta \wedge d \psi\right),
\end{aligned}
$$

which is obtained from (32) with $B=0, H=0$. The associated potential 1-form can be written as

$$
A=-\frac{Q}{2 \Sigma}\left(d t-a \sin ^{2} \theta d \phi-b \cos ^{2} \theta d \psi\right)
$$

In obtaining this expression we have gauged the potential (38) according to the transformation

$$
A=\hat{A}-\frac{Q}{2 m} d t
$$

to provide its vanishing behaviour at infinity. We shall also need the contravariant components of the electromagnetic field tensor which are given by

$$
\begin{array}{ll}
F^{t r}=\frac{Q\left(r^{2}+a^{2}\right)\left(r^{2}+b^{2}\right)}{r \Sigma^{3}}, & F^{t \theta}=\frac{Q\left(b^{2}-a^{2}\right) \sin 2 \theta}{2 \Sigma^{3}}, \\
F^{r \phi}=-\frac{Q a\left(r^{2}+b^{2}\right)}{r \Sigma^{3}}, & F^{r \psi}=-\frac{Q b\left(r^{2}+a^{2}\right)}{r \Sigma^{3}}, \\
F^{\theta \phi}=\frac{Q a \cot \theta}{\Sigma^{3}}, & F^{\theta \psi}=-\frac{Q b \tan \theta}{\Sigma^{3}} .
\end{array}
$$

Next, we shall define the electric field, as well as the dipole magnetic field in the asymptotic rest frame of the black hole. We start with the electric 1-form field $\hat{E}$ which, in the spacetime of dimensions $D$, can be defined as follows

$$
\hat{E}=-i_{\xi_{(t)}} F=(-1)^{D \star}\left(\xi_{(t)} \wedge^{\star} F\right)
$$

where $\xi_{(t)}=\xi_{(t) \mu} d x^{\mu}$ is the timelike Killing 1-form field and the $\star$ operator denotes the Hodge dual. Substituting (48) in equation (52) we obtain the following expression for the electric 1-form field in the metric (11)

$$
\hat{E}=\frac{Q}{\Sigma^{2}}\left(r d r+\left(b^{2}-a^{2}\right) \sin \theta \cos \theta d \theta\right) .
$$


The orthonormal components of the electric field in the asymptotic rest frame of the black hole are obtained by projecting (53) on the basis (25). We have

$$
\begin{aligned}
& E_{\hat{r}}=F_{\hat{r} \hat{t}}=\frac{Q}{r^{3}}+\mathcal{O}\left(\frac{1}{r^{5}}\right), \\
& E_{\hat{\theta}}=F_{\hat{\theta} \hat{t}}=\mathcal{O}\left(\frac{1}{r^{5}}\right), \\
& E_{\hat{\phi}}=F_{\hat{\phi} \hat{t}}=0
\end{aligned}
$$

We note that the dominant component of the electric field is purely radial and the associated Gaussian flux of this radial field gives the correct value for the electric charge of the black hole.

The dipole magnetic field of the black hole is described by the magnetic 2 -form defined as

$$
\hat{B}=-i_{\xi_{(t)}}{ }^{\star} F=^{\star}\left(\xi_{(t)} \wedge F\right)
$$

This can also be rewritten in the alternative form

$$
\hat{B}=\frac{1}{4} \sqrt{-g} \epsilon_{\mu \nu \alpha \beta \gamma} \xi_{(t)}^{\mu} F^{\nu \alpha} d x^{\beta} \wedge d x^{\gamma}
$$

Substituting into this expression the contravariant components of the electromagnetic field tensor (51) we obtain

$$
\begin{aligned}
\hat{B}= & \frac{Q b \sin \theta}{\Sigma^{2}}\left(r \sin \theta d r \wedge d \phi-\left(r^{2}+a^{2}\right) \cos \theta d \theta \wedge d \phi\right) \\
& +\frac{Q a \cos \theta}{\Sigma^{2}} \cos \theta\left(r \cos \theta d r \wedge d \psi+\left(r^{2}+b^{2}\right) \sin \theta d \theta \wedge d \psi\right)
\end{aligned}
$$

which in the asymptotic rest frame of the black hole has the the following orthonormal components

$$
\begin{aligned}
& B_{\hat{r} \hat{\psi}}=F_{\hat{\theta} \hat{\phi}}=\frac{F_{\theta \phi}}{r^{2} \sin \theta}=\frac{Q a}{r^{4}} \cos \theta+\mathcal{O}\left(\frac{1}{r^{6}}\right), \\
& B_{\hat{\theta} \hat{\psi}}=F_{\hat{\phi} \hat{r}}=\frac{F_{\phi r}}{r \sin \theta}=\frac{Q a}{r^{4}} \sin \theta+\mathcal{O}\left(\frac{1}{r^{6}}\right), \\
& B_{\hat{\phi} \hat{r}}=F_{\hat{\theta} \hat{\psi}}=\frac{F_{\theta \psi}}{r^{2} \cos \theta}=-\frac{Q b}{r^{4}} \sin \theta+\mathcal{O}\left(\frac{1}{r^{6}}\right), \\
& B_{\hat{\theta} \hat{\phi}}=F_{\hat{r} \hat{\psi}}=\frac{F_{r \psi}}{r \cos \theta}=-\frac{Q b}{r^{4}} \cos \theta+\mathcal{O}\left(\frac{1}{r^{6}}\right),
\end{aligned}
$$


with all others vanishing. The above expressions describe the dipole magnetic fileld created by a five dimensional weakly charged rotating black hole. We see that far from the black hole the dominating behaviour of the magnetic field is determined only by the two independent quantities

$$
\mu_{(a)}=Q a, \quad \mu_{(b)}=Q b,
$$

which can be thought of as the magnetic dipole moments of the black hole. We conclude that a weakly charged rotating black hole in five dimensions possesses two independent magnetic moments specified only in terms of the electric charge of the black hole and its two rotation parameters.

\section{GYROMAGNETIC RATIO}

It is now natural to address the gyromagnetic ratio of the five dimensional weakly charged rotating black hole we discussed above. We recall that one of the remarkable facts about a charged rotating black hole of four dimensional general relativity is that it can be assigned a gyromagnetic ratio $g=2$ just like the electron in Dirac theory [38]. The parameter $g$ is defined as a constant of proportionality in the equation

$$
\mu=g \frac{Q J}{2 M},
$$

where $M$ is the mass, $J$ is the angular momentum and $Q$ is the electric charge of the four dimensional black hole.

Turning now to the case of a weakly charged black hole in five dimensions and comparing equations (13) and (59) we see that the coupling of rotation parameters of the black hole to its mass parameter to give the specific angular momenta looks exactly the same as their coupling to the electric charge to define the magnetic dipole moments. Thus, we may write the reminiscent of equation (60) in five dimensions as follows

$$
\mu_{(i)}=\frac{Q j_{(i)}}{m}=3 \frac{Q J_{(i)}}{2 M},
$$

where we have used the relations (16) and the subscript index $i$ refers to either the parameter $a$ or, $b$. From a comparison of this equation with the classical relation (60) it becomes apparent that a five dimensional weakly charged rotating black hole can be assigned a gyromagnetic ratio $g=3$. 
Next, following the basic arguments of [28] we shall prove the value $g=3$. For this purpose, we shall define the twist [6] of a timelike Killing 1-form field $\xi_{(t)}$, which in five dimensions is the 2-form field given by

$$
\Omega=\frac{1}{2} \star\left(\hat{\xi}_{(t)} \wedge d \hat{\xi}_{(t)}\right)
$$

Physically this quantity measures the failure of the timelike Killing 1-form field to be hypersurface orthogonal. Evaluating this quantity in the metric (11) we obtain

$$
\begin{aligned}
\Omega= & -\frac{b m r}{\Sigma^{2}} \sin ^{2} \theta d r \wedge d \phi+\frac{b m\left(r^{2}+a^{2}\right)}{\Sigma^{2}} \sin \theta \cos \theta d \theta \wedge d \phi-\frac{a m r}{\Sigma^{2}} \cos ^{2} \theta d r \wedge d \psi \\
& -\frac{a m\left(r^{2}+b^{2}\right)}{\Sigma^{2}} \sin \theta \cos \theta d \theta \wedge d \psi,
\end{aligned}
$$

which implies the existence of the twist potental 1-form

$$
\omega=\frac{m}{2 \Sigma}\left(b \sin ^{2} \theta d \phi+a \cos ^{2} \theta d \psi\right)
$$

The components of this quantity in the asymptotic rest frame of the black hole show that the failure of the timelike Killing vector to be hypersurface orthogonal is completely determined by the specific angular momenta $j_{(a)}=a m$ and $j_{(b)}=b m$ of the black hole.

On the other hand, the magnetic 2-form field (57) implies the magnetic potential 1-form determined through the equation

$$
B=-d \varphi
$$

where

$$
\varphi=\frac{Q}{2 \Sigma}\left(b \sin ^{2} \theta d \phi+a \cos ^{2} \theta d \psi\right) .
$$

From this expression it follows that in the asymptotic rest frame of the black hole the magnetic potential 1-form determines the two magnetic dipole moments, just as the twist 1-form (64) determines the two specific angular momenta of the black hole. From equations (64) and (66) we read off the relation

$$
\varphi=\frac{Q}{m} \omega
$$

which, obviously, can also be rewritten in the form of (61). This proves that a five dimensional Myers-Perry black hole endowed with a small enough electric charge must have a gyromagnetic ratio of value $g=3$. The same value of gyromagnetic ratio has been found for a supersymmetric rotating black hole [39] described by the Breckenridge-Myers-Peet-Vafa (BMPV) five dimensional solution [40]. 


\section{CONCLUSIONS}

We have discussed the special properties of a five dimensional rotating black hole described by the Myers-Perry metric in the presence of an originally uniform magnetic field. The configuration of the magnetic field is supposed to have bi-azimuthal symmetry, just like the black hole spacetime itself. In this case the magnetic field has only two nonvanishing components aligned with the two angular momenta of the black hole. We have also allowed the black hole to have an electric charge small enough that the spacetime can still be described by the Myers-Perry solution.

We have constructed the 5-vector potential describing the test Maxwell field in the MyersPerry spacetime using the Killing isometries of this spacetime. The intriguing feature of this model is the appearance of non-trivial gravitomagnetic phenomena; a rotation of a five dimensional black hole in a uniform magnetic field of given configuration produces an inductive electrostatic potential difference between the event horizon and an infinitely distant surface. This potential difference comes from the superposition of two independent Coulomb fields arising due to rotations in two distinct 2-planes and two nonvanishing components of the magnetic field. Of course, in the case of an ionized medium surrounding the black hole, the potential difference will be quickly neutralized by a selective accretion process, thereby providing a mechanism for charging up the black hole.

We have also described a dipole magnetic field around a weakly charged rotating black hole in five dimensions and as expected, it turned out that the black hole possesses two independent magnetic dipole moments determined only by its electric charge, mass and angular momentum parameters. In many aspects gravitomagnetic phenomena described are qualitatively closly reminiscent of their counterparts for a four dimensional Kerr black hole immersed in a uniform magnetic field. However, there also exist some essential differences. In particular, we have shown that the gyromagnetic ratio for a five dimensional weakly charged Myers-Perry black hole is $g=3$.

In four dimensional gravity there exist stable circular orbits in the equatorial plane of a Kerr black hole. Furthermore, the presence of a uniform magnetic field around the Kerr black hole has its greatest effect in enlarging the region of stability of the circular orbits toward the horizon [41]. However, there are no stable circular orbits around a five dimensional rotating black hole, at least in the equatorial planes [20]. Therefore, it would be interesting to use 
the results of this paper to study the effect of an external magnetic field on the stability of circular motion around a five dimensional Myers-Perry metric.

\section{Acknowledgments}

A. N. thanks Gary Gibbons and Bahram Mashhoon for useful discussions on gyromagnetic ratio at an early stage of this work. V.F. thanks Feza Gürsey Institute for kind hospitality during the Research Semester Gravitation and Cosmology, June 2003. V.F is also grateful to the Natural Sciences and Engineering Research Council of Canada and the Killam Trust for their financial support.

[1] W. Israel, Phys. Rev. 164, 1776 (1967).

[2] B. Carter, Phys. Rev. Lett. 26, 331 (1971).

[3] S.W. Hawking, Commun. Math. Phys. 25, 152 (1972); D.C. Robinson Phys.Rev.Lett. 34, 905 (1975).

[4] S.W. Hawking, Commun. Math. Phys. 43, 199 (1975).

[5] V.P. Frolov and I.D. Novikov, Physics of Black Holes (Kluwer Academic Press, Dordrecht, 1998).

[6] M. Heulser, Black Hole Uniqueness Theorems (Cambridge University Press, Cambridge, England, 1996).

[7] R. Wald Quantum Fields in Curved Spacetime and Black Hole Thermodynamics (University of Chicago Press, Chicago, 1994).

[8] P. Horava and E. Witten, Nucl. Phys. B 460, 506 (1996).

[9] D. Youm, Phys. Rept. 316, 152 (1999); A. W. Peet, TASI Lectures on Black Holes in String Theory hep-th/0008241

[10] N. Arkani-Hamed, S. Dimopoulos, and D. Dvali, Phys. Lett. B 429, 263 (1998).

[11] I. Antoniadis, N. Arkani-Hamed, S. Dimopoulos, and D. Dvali, Phys. Lett. B 436, 257 (1998).

[12] L. Randall and R. Sundrum, Phys. Rev. Lett. 83, 3370 (1999).

[13] L. Randall and R. Sundrum, Phys. Rev. Lett. 83, 4960 (1999).

[14] G. Gabadadze, ICTP Lectures on Large Extra Dimensions hep-ph/0308112. 
[15] V. Rubakov, Phys. Usp. 44, 871 (2001); Usp. Fiz. Nauk 171, 913 (2001).

[16] S. Dimopoulos and G Landsberg, Phys. Rev. Lett. 87, 161602 (2001).

[17] S. B. Giddings and S. Thomas, Phys. Rev. D 65, 056010 (2002).

[18] F. R. Tangherlini, Nuovo Cimento 77, 636 (1963).

[19] R. C. Myers and M. J. Perry, Ann. Phys. (N.Y.) 172, 304 (1986).

[20] V. Frolov and D. Stojkovic, Phys. Rev. D 68, 064011 (2003).

[21] V. Frolov and D. Stojkovic, Phys. Rev. D 67, 084004 (2003).

[22] R. Emparan and H. S. Reall, Phys. Rev. Lett. 88, 101101 (2002).

[23] G. W. Gibbons, D. Ida, and T. Shiromizu, Phys. Rev. Lett. 89, 041101 (2002).

[24] G. W. Gibbons, D. Ida, and T. Shiromizu, Phys. Rev. D 66, 044010 (2002).

[25] R. Emparan and R. C. Myers, J. High Energy Phys. 0309, 025 (2003), hep-th/0308056.

[26] A. Ishibashi and H. Kodama, Prog. Theor. Phys. 110, 901 (2003), hep-th/0305185.

[27] A. Papapetrou, Ann. Inst. H. Poincare 4, 83 (1966).

[28] R. M. Wald, Phys. Rev. D 10, 1680 (1974).

[29] A. Komar, Phys. Rev. 113, 934 (1959).

[30] J. M. Bardeen, Astrophys. J. 162, 71 (1970).

[31] C. W. Misner, K. S. Thorne, and J. A. Wheeler, Gravitation, W. H. Freeman and Company (1973).

[32] A. R. King, J. P. Lasota, and W. Kundt, Phys. Rev. D 12, 3037 (1975).

[33] F. Ernst, J. Math. Phys. 17, 54 (1976).

[34] F. Ernst and W. J. Wild, J. Math. Phys. 17,182 (1976).

[35] A. N. Aliev and D. V. Gal'tsov, Phys. Usp. 32, 75 (1989); Usp. Fiz. Nauk 157, 129 (1989).

[36] B. Carter in General Relativity, An Einstein centenary survey edited by S.W. Hawking and W. Israel (Cambridge University Press, Cambridge, England, 1979).

[37] A. Chamblin, R. Emparan, and G. W. Gibbons, Phys. Rev. D 58, 084009 (1998).

[38] B. Carter, Phys. Rev. D 174, 1559 (1968).

[39] C. A. R. Herdeiro, Nucl. Phys. B 582, 363 (2000).

[40] J. Breckenridge, R. Myers, A. Peet, and C. Vafa, Phys. Lett. B 391, 93 (1997).

[41] A. N. Aliev and N. Özdemir Mon. Not. R. Astron. Soc. 336, 241 (2002), gr-qc/0208025.

[42] In the brane world scenario, when charged particles can live only on the brane, this conclusion also remains true. In the presence of a plasma of charged particles located on the brane there 
will be a selective accretion process reducing the charge $\tilde{Q}$. 